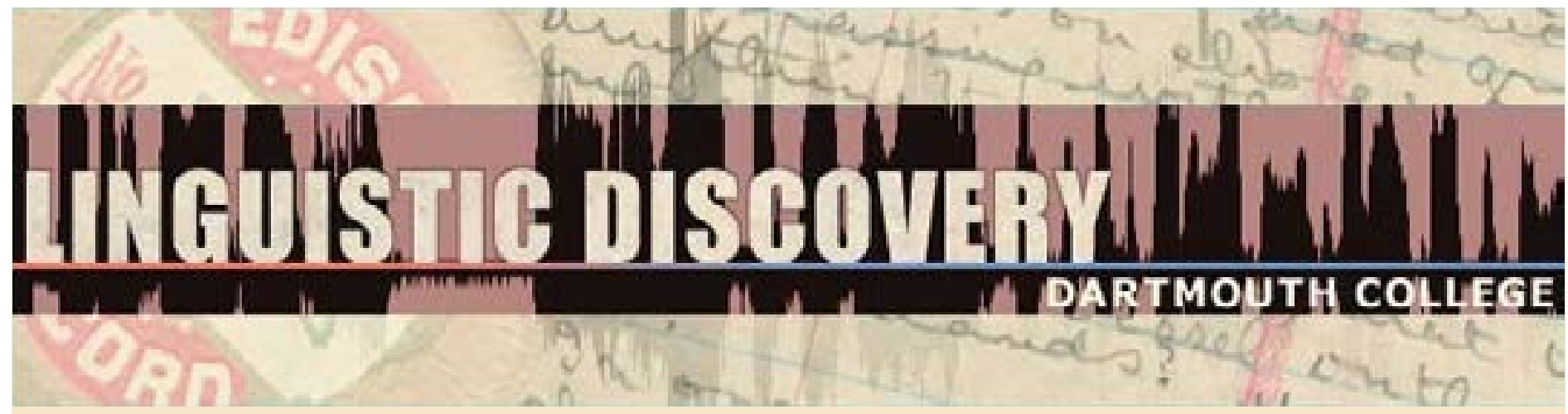

Volume 5

Issue 1 2007
Ngbugu Digital Wordlist: Presentation Form

Kenneth S. Olson

SIL International and University of North Dakota

Jacques Vermond Mbomate L'Association Centrafricaine pour la Traduction de la Bible et D'Alphabétisation

doi: 10.1349/PS1.1537-0852.A.317

url: http://journals.dartmouth.edu/cgi-bin/WebObjects/ Journals.woa/1/xmlpage/1/article/317
Linguistic Discovery

Published by the Dartmouth College Library Copyright to this article is held by the authors. ISSN 1537-0852 linguistic-discovery.dartmouth.edu 


\title{
Ngbugu digital wordlist: Presentation form
}

\author{
Kenneth S. Olson \\ SIL International and University of North Dakota
}

\section{Jacques Vermond Mbomate \\ L'Association Centrafricaine pour la Traduction de la Bible et d'Alphabétisation}

This paper presents a 204-item digital wordlist of Ngbugu, an Ubangian language spoken in Central African Republic. The wordlist includes orthographic and broad phonetic transcriptions of the words, French and English glosses, an individual WAV recording of each word, GIF images of the original field transcriptions, and metadata for resource discovery. This presentation form of the wordlist was generated from an archived version (Olson 2006) following the procedure laid out in Simons, Olson and Frank (2007).

\section{Introduction}

This paper presents a 204-item digital wordlist of core vocabulary in Ngbugu, an Ubangian language spoken in Central African Republic by approximately 95,000 people (ISO 639-3 code: [lnl], Gordon 2005). The materials included in this presentation of the data include the following:

-Wordlist: this interactive webpage, containing orthographic and broad phonetic transcriptions of each word, as well as French and English glosses.

- Recordings: WAV digital recordings of each word, accessible by clicking on the orthographic form of each word in the list below. Your web browser will attempt to play the recording with the sound program that is set up as the default WAV player on your system. The recordings were made with a sample rate of $44,100 \mathrm{~Hz}$ and a quantization of 16 bits.

-Field transcriptions: GIF images of the handwritten field transcriptions of the data.

- Metadata: a resource description of the data. This is useful for resource discovery, for example with the Open Language Archives Community (OLAC) [http://www.language-archives.org]

The original wordlist materials include two items: a two-page paper wordlist form and a 16minute audio cassette recording. The wordlist form presents the standardized wordlist of 204 items from Moñino (1988). For each item the form provides a prompt in French and a space for the transcription of the elicited form. The form was filled in with handwritten Ngbugu orthographic transcriptions by the second author, a Ngbugu speaker literate in both Ngbugu and French. Some items included a suggested alternative pronunciation in parentheses or an indication of uncertainty concerning the data. The first author verified the list in consultation with the second author, and together they produced a broad phonetic transcription employing the International Phonetic Alphabet (IPA 1999).

The first author then created a revised list in Microsoft Word for Windows 2000 that included the French prompt, the orthographic rendering, and the IPA rendering. The accompanying audio cassette contains a recording of the second author repeating this revised list. He produced the French prompt first, then the corresponding Ngbugu form. The recording was 
made with a Marantz PMD 420 monaural cassette recorder and an Audio-Technica ATM 33a microphone. The recording session took place on March 6, 2004, at the ACATBA center (l'Association Centrafricaine pour la Traduction de la Bible et d'Alphabétisation) in Bangui, Central African Republic.

The process by which this field transcription and audio data were converted into digital forms suitable for long-term archiving is discussed in Simons, Olson, and Frank (2007). That paper also describes how this presentation form was generated from the archival form. The complete archival recording (Olson 2006) can be ordered from:

SIL Language and Culture Archives

7500 W Camp Wisdom Rd

Dallas TX 75236 USA

archive_dallas@sil.org

\section{The Wordlist}

See complete resource description.

See field transcriptions:

Page 1 (items 1 - 99)

Page 2 (items 100 - 204)

\begin{tabular}{|c|c|c|c|c|}
\hline French gloss & English gloss & $\begin{array}{l}\text { orthographic } \\
\text { transcription }\end{array}$ & $\begin{array}{l}\text { phonetic } \\
\text { transcription }\end{array}$ & \\
\hline abeille & bee & wräto & [wьātò] & \\
\hline acide (vb) & $\operatorname{tart}$ & kpï; kï & {$[\mathrm{kp} \overline{1}]$} & \multirow[t]{3}{*}{ (adjective) } \\
\hline aile & wing & $\underline{\text { mbrö }}$ & [mbsō] & \\
\hline aller & go & $\underline{\mathrm{e}}$ & [?è] & \\
\hline amer (vb) & bitter & $\underline{\text { chü }}$ & {$\left[\int \overline{\mathrm{u}}\right]$} & \multirow[t]{6}{*}{ (adjective) } \\
\hline animal & animal & gia & [già] & \\
\hline année & year & ngû & [ngú] & \\
\hline appeler & call & e tchô & [?è tfó] & \\
\hline arbre & tree & yö & {$[\mathrm{jo}]$} & \\
\hline attacher; lier & attach & $\underline{\mathrm{i}(\mathrm{reu})}$ & [?i] & \\
\hline blanc & white & grô & [gьо́] & \multirow[t]{4}{*}{ (adjective) } \\
\hline boire & drink & $\underline{\text { ndjo }(\mathrm{reu})}$ & [ndzò] & \\
\hline bon & good & tchô & [tfó] & \\
\hline & & tchôtchô & 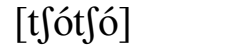 & \\
\hline bouche & mouth & $\underline{\mathrm{ma}}$ & [mà] & \multirow{4}{*}{$\begin{array}{l}\text { (arm) } \\
\text { (hand) }\end{array}$} \\
\hline bras/main & $\mathrm{arm} / \mathrm{hand}$ & könô & [kōnó] & \\
\hline & & tchâneû & [tfánó] & \\
\hline brouillard & mist; fog & $\underline{\text { ndrö }}$ & [ndsō] & \\
\hline
\end{tabular}


17. brûler (intr.) burn (intr.) ju [3ù]

18. brûler (trans.) burn (tr.) [ cho

19. ceci this

20. celà that

21. ce (en question) this

22. cendres

23. champ

24. charbon

25. chaud/chauffer

26. chemin

27. chèvre

28. chien

29. chose

30. cinq

31. Coeur

32. colline/termitière

33. compter

34. connaître

35. corde

36. corne

37. cou/gorge

38. couper (couteau)

39. couper (hache)

40. cracher

41. creuser

42. cuire

43. cuisse

44. cul; fondement

45. cultiver

46. danser

47. debout (être)

48. dent

49. deux

50. dire

51. donner

52. dormir

53. droite (à)

54. eau

55. écorce

56. elephant

57. enfant

\begin{abstract}
his
\end{abstract}
hat

ash

garden

charcoal

hot/heat

path

goat

dog

thing

five

heart

hill/termite hill

count

know

cord

horn

neck/throat

cut

chop

spit

dig

cook

thigh

bottom

cultivate

dance

stand

tooth

two

say

give

sleep

right

water

bark

child elephant

geö [a하 aneü âteu [ànō átò]

aneü lâme [ànō lámè]

reu âteu [вว̀ átò]

vöreu [vōธò]

keöndeö $\quad[\mathrm{k \imath nd}$

mbüreu [mbūbò]

awö [àwō]

wo [wò]

köbo [kōbò]

ëbro [ēbsò]

ëvrô [ēvьó]

reu [Бว̀]

mïndûu [mīndû]

$\underline{\text { su } \quad \text { [sù] }}$

koto [kòtò] (hill)

koto asâko [kòtò àsákò] (termite hill)

deu (reu) [dò вə̀]

wunseû [wùsó]

$\underline{w \hat{u}}$ [wú]

$\underline{\mathrm{du}} \quad[\mathrm{dù}]$

wa [wà]

de [dè]

kuteû [kùtó]

di [di]

fa [fà]

kü $\quad[\mathrm{ku}]$

kpâ bêche [kpá béfè]

djo (keöndeö) [dzò kı̀nd̄̄]

da ëngbä [dà ēngbā]

kro lafô $\quad$ [kьò làfó]

¡ii [3ī]

bïchuû [bî̀ùu

to pö [tò pō]

za (reu seü) [zà ьә̀ sā]

lo (lö) [lò]

deu ndôkö [dò ndókō]

ngûu [ngú]

kongôâa [kòngóá]

mbala [mbàlà]

bechë [bè (hot (adj.))

(heat (vb.)) 


\begin{tabular}{|c|c|c|c|c|c|}
\hline 58. & enfanter & give birth & zeo bechë & [zì bè)ēe] & \\
\hline 59. & enfler & swell & mera & [тѐьà] & \\
\hline & & & $\underline{\underline{\text { suku }}}$ & [sùkù] & \\
\hline 60. & entendre & heart & dje (pö) & [dzè pō] & \\
\hline 61. & envoyer & send & woan & [woã] & \\
\hline 62. & épine & thorn & chi & {$[\mathrm{Si}]$} & \\
\hline 63. & étoile & star & kêkêreû & [kékéвә́] & \\
\hline 64. & étranger & stranger & yireu & [јі̀кว̀] & \\
\hline 65. & excréments & excrement & kändjâ & [kāndzá] & \\
\hline 66. & façonner (pot) & $\begin{array}{l}\text { make (clay } \\
\text { pot) }\end{array}$ & $\underline{\text { soa }}$ & [soà] & \\
\hline 67. & fagot & firewood & $\underline{\text { ëwo }}$ & [ēwò] & \\
\hline 68. & faim & hunger & go & [gò] & \\
\hline 69. & farine & flour & zûwü & [zúwū] & $\begin{array}{l}\text { (alt: zûü } \\
\text { (zúū)) }\end{array}$ \\
\hline 70. & femme & female & ëchë & {$[\overline{\mathrm{e}} / \overline{\mathrm{e}}]$} & \\
\hline 71. & fer & iron & kwö & {$[\mathrm{kwō}]$} & \\
\hline 72. & feu & fire & wo & [wò] & \\
\hline 73. & feuille & leaf & kökô & [kōkó] & \\
\hline 74. & filet & net & gbânda & [gbándà] & \\
\hline 75. & finir & finish & $\mathrm{ka}(\mathrm{reu})$ & [kà] & \\
\hline 76. & fleche & arrow & wûlî & [wúlí] & \\
\hline 77. & foie & liver & $\overline{b e}$ & [bè] & \\
\hline 78. & forger & forge & mbo ndawo & [mbò ndàwò] & \\
\hline 79. & frapper; batter & hit & $\overline{\text { bhi }}$ & [6i] & \\
\hline 80. & froid (être) & cold & $\overline{\mathrm{zeo}}$ & [Z̈̈] & \\
\hline 81. & fructifier & bear fruit & le & [lè] & \\
\hline 82. & fumée & smoke & ngôwo & [ngówò] & \\
\hline 83. & gauche (à) & left & deu gele & [dà gèlè] & \\
\hline 84. & genou & knee & $\overline{\text { köta }}$ & [kōtà] & \\
\hline 85. & graisse/huile & fat/oil & jôrââ & [зо́ка́] & (fat) \\
\hline & & & kâdâ & [kádá] & (oil) \\
\hline 86. & grand & big & gbölö & [gbōlō] & \\
\hline 87. & gratter (se) & scrape & ngro teû & [ngsò tó] & \\
\hline 88. & griller & grill & $\mathrm{zo}(\mathrm{reu})$ & [zò ธà] & \\
\hline 89. & guerre & war & kwö & {$[\mathrm{kw} \overline{\mathrm{o}}]$} & \\
\hline 90. & herbe & grass & bë & {$[\mathrm{be}]$} & \\
\hline 91. & homme & male & köchë & {$\left[\mathrm{k} \bar{o} \int \overline{\mathrm{e}}\right]$} & \\
\hline 92. & hyène & hyena & gbongô & [gbòngó] & \\
\hline 93. & il & he/she & tche & {$\left[\mathrm{t} \int \hat{\mathrm{e}}\right]$} & \\
\hline & & & tchetchë & [tfetsè] & \\
\hline 94. & ils & they & andjề & [àndzé] & \\
\hline 95. & intestines & intestines & chêtê & [Sété] & \\
\hline
\end{tabular}


96. je

97. jumeaux

98. lancer; jeter

99. langue (organe)

100. laver

101. long

102. lourd

103. lune

104. maison

105. manger

106. marche/marcher

107. mauvais (être) bad

108. mere

109. montagne

110. mordre

111. mortier

112. mouche

113. mourir

114. ne...pas

115. neuf; nouveau

116. nez

117. noir

118. nom

119. nombreux (être)

120. nombril

121. nous

122. nuit

123. oeil/visage

124. oeuf

125. oiseau

126. oncle (maternel)

127. ongle/griffe

128. Oreille

129. os

130. panthère
I më [mē]

twins

amêä

[àméā]

[àngbòlà]

(for

animals)

$\begin{array}{ll}\text { yi reu } & {[\text { [ì sò] }} \\ \underline{\text { tïmï }} & {[\text { tìmī] }} \\ \underline{\text { djutu }} & {[\text { dzùtù] }} \\ \underline{\text { ngbongro }} & \text { [ngbòngrò] } \\ \underline{\text { keülû }} & {[\text { kōlú }]}\end{array}$

(alt: külû reu

(kūlú ธə̀))

nyîpï [nípī]

nda [ndà]

zeo reu [ż̀ เò]

neû [nó]

neu [nò]

ngbongbo [ngbòngbò]

amâ [àmá]

anyï [ànī]

aga [àgà]

lo $\quad[$ lò]

frö $\quad[\mathrm{f} b \overline{0}]$

vömä [vōmā]

tchu [tfù]

ấbo [ábò]

ngbreôndjï [ngbڤîndzīi] (alt:

ngbrôndjï

(ngbьóndzī))

[wũ]

$\begin{array}{ll}\underline{\text { bübü }} & {[\mathrm{bū} b \bar{u}]} \\ \text { reü } & {[\mathrm{s} \overline{]}]}\end{array}$

ngbäkotche [ngbākòt $\left.\int e\right]$

ngawun [ngàwũ]

azeû [àzó]

tchîleû bütchô [tfíló būtfó]

la [là] (eye)

tchâtchu [tfátfù] $\quad$ (face)

prö [рьо̄]

nyiëneü [nīēnō]

bird

chïmbï

tiu

gbäbï

[] (no form)

[ $\overline{\mathrm{i}} \mathrm{mb} \overline{\mathrm{i}}]$

[tū]

[gbābīi]

[mí́ś] 


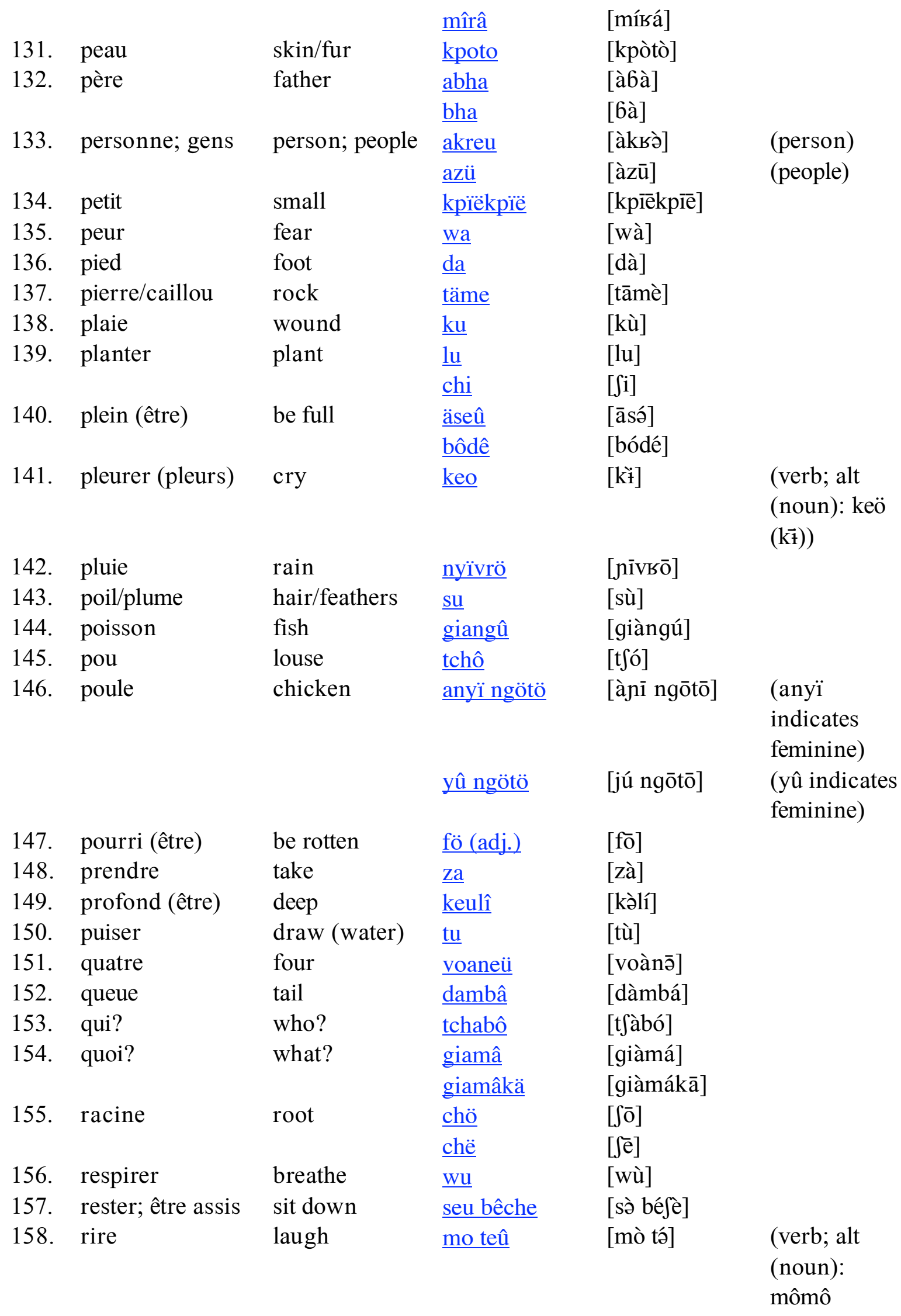


159. rosée

160. rouge

161. sable

162. sagaie

163. saison des pluies

164. saison seche

165. salive

166. sang

167. sec (être)

168. sein

169. sel

170. semence/grain

171. sentir (intr.)

172. serpent

173. soleil

174. souffler

175. sucer

176. sucré (être)

177. termite

178. terre; sol

179. tête

180. téter

181. tomber

182. tortue

183. tous; tout

184. tousser

185. tranchant (être)

186. transpercer

187. travail

188. trios

189. trou

190. tu

191. tuer

192. un

193. urine

\begin{abstract}
Dew
\end{abstract}
red

sand

spear

rainy season

dry season

saliva

blood

dry

breast

salt

seed/grain

smell

snake

sun

blow

suck

be sweet

termite

earth; soil

head

nurse

fall

turtle

all

cough

be sharp

pierce; stab

work

three

hole

you (2sg)

kill

one

urine

\begin{tabular}{|c|c|}
\hline ngômëä & [ngómēāā \\
\hline ngômë & [ngómē] \\
\hline ngbê & [ngbé] \\
\hline woye & [wòjè] \\
\hline deö & {$[\mathrm{d} \overline{\mathrm{t}}]$} \\
\hline zöngö & [zōngō] \\
\hline ngbeôgeô & [ngbîgît] \\
\hline ngrôsö & [пяьо́sō] \\
\hline & \\
\hline
\end{tabular}

[ngò]

[ngbьï]

[ngbьò]

[ngoà]

[ndzí]

[tfáfò]

[ēkьó]

[lò]

[?ธò]

[?à]

[à tfōo]

[bò]

[Sò]

[kūmà]

[?à ngò]

[tè]

[kūlú]

[kūlá]

[gbé]

[tèkò]

[kì]

[sù]

[kьò]

[kuà]

[vōtàá]

[kùdú]

[mòmō]

[wõ]

[bàlē]

[ngándī] (mómó))

(also used

for a sterile

woman)

(seed)

(grain) 


\begin{tabular}{|c|c|c|c|c|}
\hline 194. venir & come & neu atâ & [nò àtá] & $\begin{array}{l}\text { (alt: neu } \\
\text { atêteu (nò } \\
\text { àtétò)) }\end{array}$ \\
\hline vent & wind & yi & [jì] & \\
\hline ventre & abdomen & $\underline{\mathrm{vu}}$ & {$[\mathrm{v} \overline{\mathrm{u}}]$} & \\
\hline viande/chair & meat/flesh & gûsû & [gúsú] & (meat) \\
\hline & & mbë & {$[\mathrm{mbe \overline {e }}]$} & (flesh) \\
\hline village & village & gö & {$[g \overline{0}]$} & \\
\hline voir & see & wun & [wũ] & \\
\hline voler (oiseau) & fly & wro & [wьò] & \\
\hline voler; dérober & steal & zeo ngbä & [ż̀ ngbā] & \\
\hline vomir & vomit & ndjrateû & [ndzкà tó] & \\
\hline vouloir & want & nyindeû & [kó jìndó] & \\
\hline vous & you (2pl) & nyë & {$[\mathrm{ne}]$} & \\
\hline
\end{tabular}

\section{References}

Gordon, Raymond G. (ed.) 2005. Ethnologue: Languages of the world. 15th edition. Dallas: SIL. [http://www.ethnologue.com].

International Phonetic Association. 1999. Handbook of the International Phonetic Association. Cambridge: Cambridge University Press.

Moñino, Yves (ed.) 1988. Lexique comparative des langues oubanguiennes. Paris: Geuthner.

Olson, Kenneth S. 2006. Ngbugu digital wordlist: Archival form. SIL-LCA-47050. SIL Language and Culture Archives, Dallas, Texas.

Simons, Gary F.; Kenneth S. Olson; and Paul Frank. 2007. Ngbugu digital wordlist: A test case for best practices in archiving and presenting language documentation. Linguistic Discovery 5.1:28-39. Online. URL: [http://journals.dartmouth.edu/cgibin/WebObjects/Journals.woa/2/xmlpage/1/article/314]

Authors' contact information:

Kenneth S. Olson

SIL

7500 W Camp Wisdom Rd

Dallas, TX 75236

http://www.sil.org/ olsonk

Jacques Vermond Mbomate

L'Association Centrafricaine pour la Traduction de la Bible et d'Alphabétisation

B.P. 1990

Bangui

Central African Republic

Jacques_Mbomate@wycliffe.net 